Cite this: Phys. Chem. Chem. Phys., 2014, 16, 11265

Received 2nd April 2014, Accepted 23rd April 2014

DOI: $10.1039 / c 4 c p 01429 d$

www.rsc.org/pccp

\title{
Molecular self-assembly at nanometer scale modulated surfaces: trimesic acid on Ag(111), $\mathrm{Cu}(111)$ and $\mathrm{Ag} / \mathrm{Cu}(111) \dagger$
}

\author{
Mahdi Sadeghzadeh Baviloliaei and Lars Diekhöner*
}

\begin{abstract}
The balance between molecule-molecule and molecule-surface interactions is a determining factor in the creation of well-ordered organic networks formed by self-assembly on crystalline metal surfaces. We have used a scanning tunneling microscope under ultrahigh vacuum conditions to study the molecular selfassembly of trimesic acid on a surface that is modulated on a comparable nanometer scale as the size of the molecules. This is made of one layer of silver grown on a $\mathrm{Cu}(111)$ surface where it forms a periodic reconstruction. It is shown that the self-assembly of trimesic acid at room temperature, where intermolecular interactions are taking place via hydrogen bonds, is strongly disturbed due to the modulated substrate and the spatially varying potential imposed on the molecules. Annealing to $350 \mathrm{~K}$ partly deprotonates the molecules and changes the intermolecular interactions to stronger ionic hydrogen bonds. This reduces the influence of the modulated substrate and allows the molecules to self-assemble into long-range ordered networks on the surface. Comparisons are made to self-assembly on the flat surfaces of $\mathrm{Ag}(111)$ and $\mathrm{Cu}(111)$, where we always find well-ordered molecular networks.
\end{abstract}

\section{Introduction}

Molecular interactions at surfaces play an important role in many fields such as molecular electronics, heterogeneous catalysis, biocompatibility or sensor technology. Understanding the interaction between molecules and surfaces as well as the inter-molecular interactions on the surface is therefore crucial. For many purposes it is of advantage to assemble molecules in periodic networks covering the whole surface. This can be achieved by using organic molecules, which are allowed to self-assemble on the surface. ${ }^{1-5}$ The properties can be tailored by choosing the right functionality of the molecules. Naturally the physical shape of the molecules and the directionality of the bonds involved will influence the overall geometry of the molecular networks. A delicate balance between molecule-surface bonding, inter-molecular interactions and kinetic parameters like temperature and deposition rate will decide on the shape of the formed network. In this article we will focus on the role of the substrate ${ }^{6-11}$ by using crystalline metal surfaces that are either flat or given a nanometer scale modulation beforehand. For metal growth, it is well-known that the symmetry of the surface strongly influences the geometry of

Aalborg Universitet, Institut for Fysik og Nanoteknologi, Skjernvej 4a, 9220 Aalborg, Denmark.E-mail:ld@nano.aau.dk

$\dagger$ Electronic supplementary information (ESI) available: Additional STM images and molecular models of TMA on Cu(111) after annealing. See DOI: 10.1039/ c4cp01429d the structures grown on top. ${ }^{12}$ But organic molecules typically have a length scale (nanometer) which is much larger than the atomic periodicity of low-index metal surfaces (few Å). Therefore we have used a surface which has been given a nanoscale periodic modulation and investigate its influence on the molecular interactions and the self-assembly.

Trimesic acid (TMA) consists of a phenyl ring with three carboxylic acid groups. It has been studied on crystalline surfaces like $\mathrm{Cu}(100), \mathrm{Ag}(111)$ and $\mathrm{Au}(111)^{13-16}$ under ultrahigh vacuum conditions, where it interacts via hydrogen bonding and forms well-organized structures by self-assembly. ${ }^{17}$ TMA forms an open honey-comb phase at room temperature on the noble metal surfaces $A g(111)$ and $A u(111)$. A rich variety of structures are observed upon increasing coverage on $\mathrm{Au}(111)^{16}$ and on $\mathrm{Ag}(111)$ the TMA honey-comb phase is transformed into a close-packed phase upon annealing to $420 \mathrm{~K} \cdot{ }^{14,15}$ This is associated with a deprotonation of one carboxylic acid group of the TMA that allows interaction via stronger ionic hydrogen bonds between the negatively charged oxygen of the acid group and a neighbouring TMA. ${ }^{15,18,19}$ Studies on $\mathrm{Cu}(100)$ showed that the ordered domains are significantly smaller due to the stronger molecule-surface interaction on the more reactive $\mathrm{Cu}(100)$ surface. ${ }^{13}$ Also here a range of structures are observed depending on coverage and it was studied how the deprotonation depends on coverage and annealing temperature. ${ }^{20}$ Some of the TMA molecules are bonded to the surface in an upright standing configuration on $\mathrm{Cu}(100) .{ }^{13}$ These molecules appear in a round shape in the 
scanning tunneling microscope images while the flat lying TMA molecules are imaged in a triangular shape, as seen on $\mathrm{Ag}(111)$ and $\mathrm{Au}(111)^{13-16}$ and in the work presented here.

The modulated substrate we use here consists of one monolayer of $\mathrm{Ag}$ grown on the surface of $\mathrm{Cu}(111)$. Due to a lattice mismatch between $\mathrm{Ag}$ and $\mathrm{Cu}$, this forms the well-known $(9 \times 9)$ reconstruction, with a periodicity of $2.4 \mathrm{~nm}$ and a corrugation on the order of $0.25 \AA^{21-24}$ These kind of weak reconstructions and Moiré patterned surface systems have been seen for many materials. $^{25-30}$ We will compare the self-assembly of TMA on this modulated surface with the flat surfaces of $\operatorname{Ag}(111)$ and $\mathrm{Cu}(111)$. Changing the balance between molecule-molecule and molecule-surface interaction is shown to have a drastic effect on the self-assembly: we will demonstrate that the molecular self-assembly of TMA at room temperature is strongly disturbed due to the modulated substrate as long as TMA is interacting via fairly weak hydrogen bonding, whereas annealing allows a stronger intermolecular interaction via ionic hydrogen bonds that leads to a new well-ordered phase with shape and symmetry steered by the substrate.

\section{Experimental}

All experiments have been performed under Ultra High Vacuum (UHV) conditions (base pressure of $1 \times 10^{-10} \mathrm{mbar}$ ) using a scanning tunneling microscope (STM). The metal samples have been cleaned by sputtering and subsequent annealing to $800 \mathrm{~K}$. TMA molecules have been deposited by sublimation from a Knudsen cell and silver metal films have been prepared using an e-beam evaporator. All coverages in monolayers (ML) below are given in terms of how large an area fraction the molecules cover. All STM images have been taken with the sample at room temperature (RT).

\section{Results and discussion}

\section{TMA on $\mathrm{Ag}(111)$ and $\mathrm{Cu}(111)$}

TMA on Ag(111) has previously been studied at RT and $420 \mathrm{~K}^{14,15}$ Fig. 1 shows a typical STM image of TMA on Ag(111) prepared and scanned at RT. We observe that more than $90 \%$ of the covered surface is filled with TMA in the honey-comb phase. Annealing the surface to $420 \mathrm{~K}$ leads to the formation of a close-packed phase. The transformation occurs through in-plane compression and deprotonation of the carboxylic acid functional groups. ${ }^{15} \mathrm{We}$ find a density of 0.9 Molecules per $\mathrm{nm}^{2}$ (M per $\left.\mathrm{nm}^{2}\right)$ in the honey-comb phase and 1.44 $\mathrm{M}$ per $\mathrm{nm}^{2}$ in the close-packed phase.

Next we present the self-assembly of TMA on $\mathrm{Cu}(111)$. Fig. 2a shows the STM image of 0.7 ML TMA deposited at room temperature on $\mathrm{Cu}(111)$. The TMA molecules are always imaged in a triangular form and are therefore flat lying on the surface at all surface temperatures we investigated. We observe a high degree of order with mostly honey-comb structures with the same bonding distance and periodicity as on $\mathrm{Ag}(111)$ and a density of $0.9 \mathrm{M}$ per $\mathrm{nm}^{2}$. Some parts (around 20\%), of the surface are covered with closer packed structures with a density

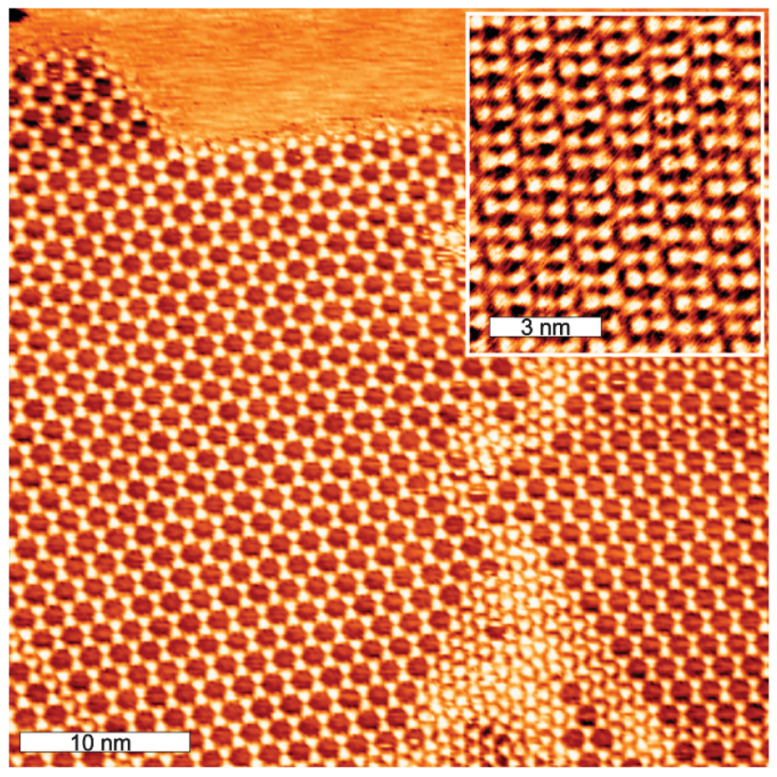

Fig. 1 TMA on $A g(111)$ at RT. The inset shows the close-packed phase after annealing to $420 \mathrm{~K}$.

of 1.4 M per $\mathrm{nm}^{2}$ (Fig. 2b). It should be noted that this phase is different from the close-packed phase we observe after annealing as presented in the following.

Upon annealing to higher temperatures the honey-comb phase disappeared and we only observed a high-density closepacked phase. This transformation happens already at $325 \mathrm{~K}$. This is in contrast to $\mathrm{Ag}(111)$, where it is necessary to anneal to $420 \mathrm{~K}$ in order to transform all molecules into close-packed assemblies. ${ }^{15}$ Fig. $2 \mathrm{c}$ and d shows two kinds of arrangements after annealing: single-row and double-row close-packed formations. We find $40 \%$ in single- and $60 \%$ in double-rows. Both the singleand double-row structures are packed with a density of $2.0 \mathrm{M}$ per $\mathrm{nm}^{2}$. In the ESI $\dagger$ we show detailed STM images of both structures including molecular models. This is $43 \%$ higher than the closepacked phase observed after deposition (Fig. 2b) and 40\% higher than the density of the close-packed phase on $\operatorname{Ag}(111)$. We speculate that this is due to, that the TMA molecules have lost more than one proton on the more reactive $\mathrm{Cu}$ surface allowing a closer interaction mediated by an increased number of ionic hydrogen bonds compared to $\mathrm{Ag}(111)$, where only one of the three carboxylic acid groups are deprotonated. ${ }^{15}$ We furthermore note, that the annealing to $325 \mathrm{~K}$ allows TMA molecules to descend-ascend steps between terraces, since we observed some terraces to be empty of molecules and neighbouring terraces to be filled with molecules. Further annealing (up to $420 \mathrm{~K}$ was tried) did not change the structures.

\section{TMA on modulated $\mathrm{Ag} / \mathrm{Cu}(111)$}

The modulated substrate was prepared by evaporating $1 \mathrm{ML}$ of $\mathrm{Ag}$ on the $\mathrm{Cu}(111)$ surface at room temperature. The periodicity of the $(9 \times 9) \mathrm{Ag} / \mathrm{Cu}(111)$ layer is $2.4 \mathrm{~nm}^{.21-24}$ Fig. 3a shows an STM image of sub-monolayer amounts of TMA deposited on $\mathrm{Ag} / \mathrm{Cu}(111)$ at room temperature. We observe an intermixed 


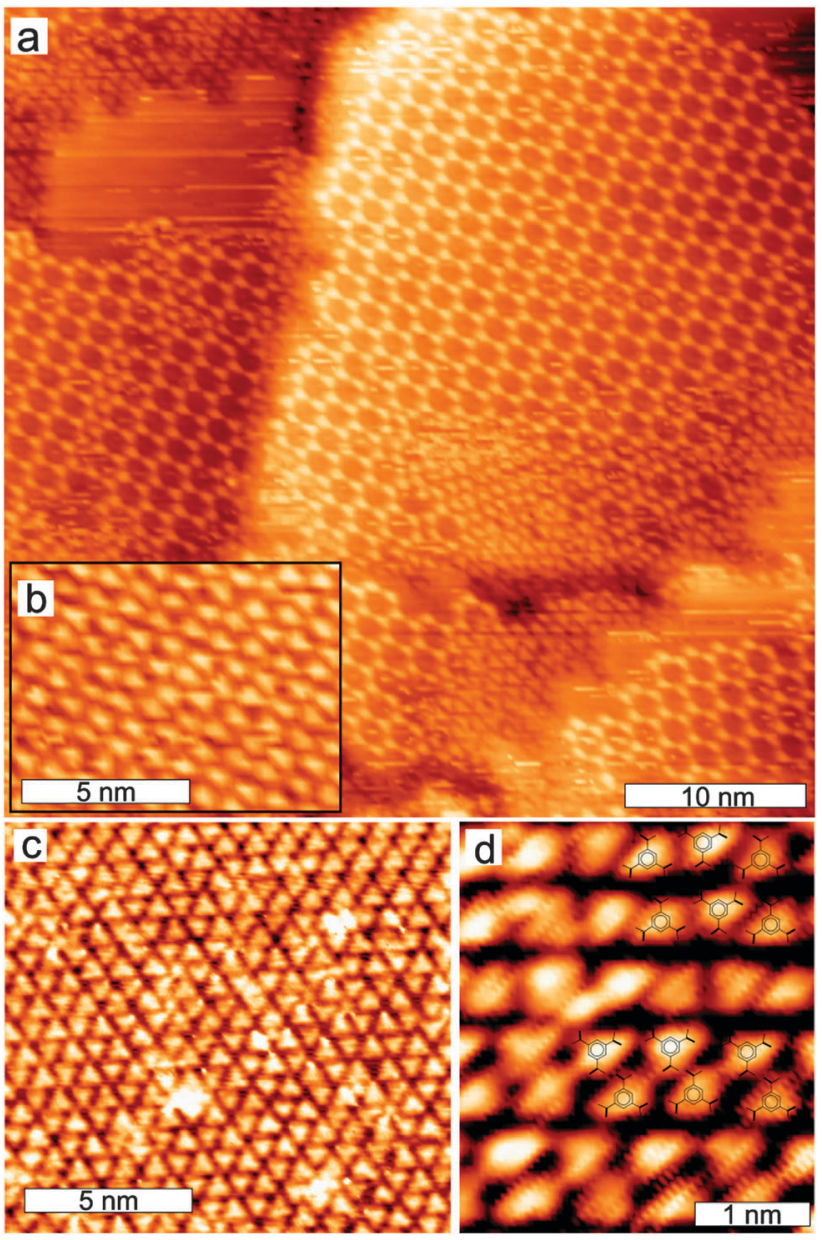

Fig. 2 (a) STM image of TMA adsorbed on $\mathrm{Cu}(111)$ at room temperature. We observe domains with honey-comb, close-packed and empty $\mathrm{Cu}(111)$ areas. (b) Close-up image of the "low-density" compact structure at room temperature. (c) STM image of the "high-density" phase of TMA on Cu(111) adsorbed at room temperature followed by annealing to $325 \mathrm{~K}$. All TMA molecules are imaged triangular and are therefore flat-lying. (d) Highresolution image of the $\mathrm{TMA} / \mathrm{Cu}(111)$ after annealing to $325 \mathrm{~K}$ illustrating the two kinds of row-formations: the upper 3 rows are single-row structures and the lower 4 rows are two pairs of double-row structures. Molecular models of TMA are superimposed.

phase consisting of regular honey-comb units, honey-comb units that are filled with a TMA molecule inside and patches with closer packed TMA molecules. The most important observation is the lack of long-range order. In the upper part of the image the modulated $\mathrm{Ag} / \mathrm{Cu}(111)$ substrate is visible. Fig. $3 \mathrm{~b}$ shows a high-resolution STM image, where it can be seen that the filled honey-comb structures have the same size as the unfilled. Furthermore, the distance between adjacent honeycomb units does not depend on the filling. Comparing sizes and inter honey-comb distances we measure the same values on $\mathrm{Ag} / \mathrm{Cu}(111)$ as on $\mathrm{Cu}(111)$ and $\mathrm{Ag}(111)$. The center-filling TMA molecule is placed exactly in the middle of a honey-comb and it is interacting via hydrogen bonds between each of the 3 carboxyl groups and the 3 closest TMA's as illustrated in the model picture in Fig. 3c. These center-filled honey-comb structures are an example of host-guest architectures that play an
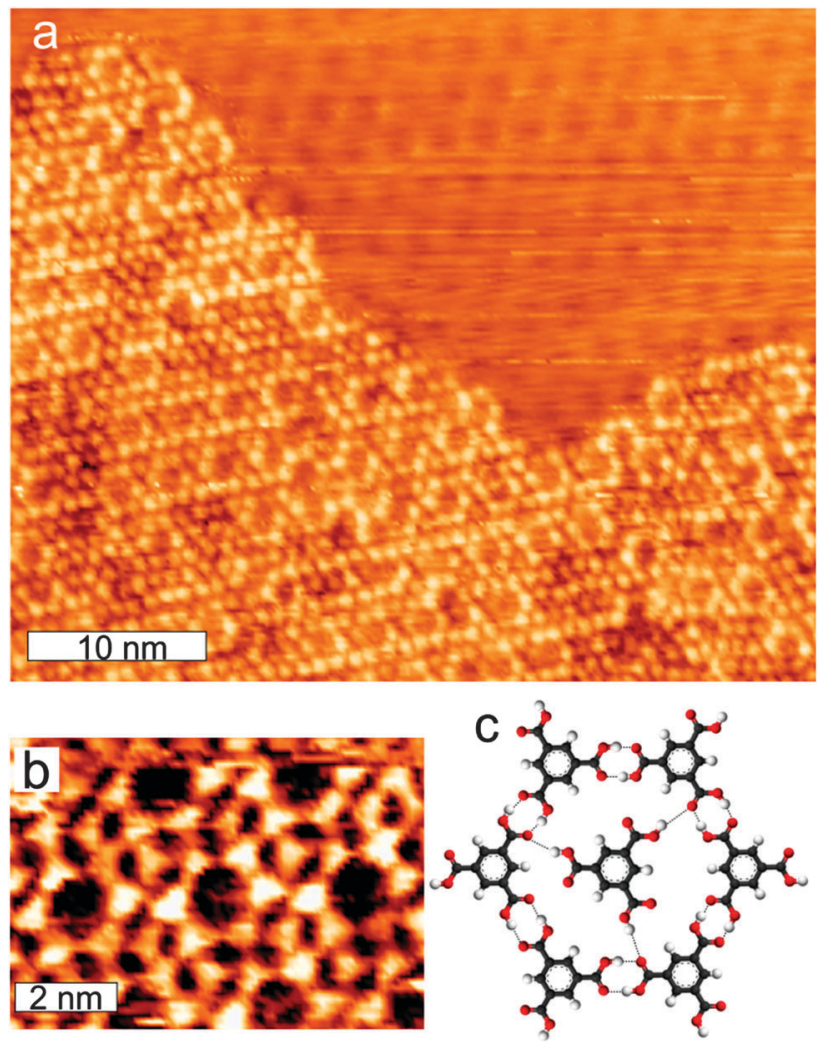

Fig. 3 (a) STM image of TMA adsorbed at room temperature on $1 \mathrm{ML} \mathrm{Ag/}$ $\mathrm{Cu}(111)$. The modulated substrate is visible in the upper right part of the image. (b) Close-up STM image showing an area with honey-comb structures, where some of the units are center-filled with a TMA molecule. (c) An illustration of a center-filled honey-comb hexagon. The dotted lines indicate the presumed $\mathrm{H}$-bonding

important role in surface chemistry e.g. and has been observed previously for other systems, for example TMA on graphite. ${ }^{31}$ Due to the larger honey-comb size of TMA on graphite the center-filling TMA was believed to be placed acentric. ${ }^{31}$

Annealing the sample to $350 \mathrm{~K}$ led to a compression and formation of a well-ordered close-packed phase, where two TMA molecules pair up as it was also seen on $\operatorname{Ag}(111)$. This is shown in Fig 4a, where the TMA pairs are observed on the right hand side and the $\mathrm{Ag} / \mathrm{Cu}(111)$ substrate on the left hand side of the image. We believe that a deprotonation has taken place, like on $\operatorname{Ag}(111) \cdot{ }^{15}$ Interestingly our studies showed that the transformation to the close-packed phase already can happen at lower annealing temperatures compared with the $\mathrm{Ag}(111)$ surface, where temperatures of $420 \mathrm{~K}$ are required to fully convert the TMA molecules into a close-packed structure. ${ }^{15}$ We believe that $\mathrm{Ag} / \mathrm{Cu}(111)$ has a higher chemical reactivity that facilitates the necessary deprotonation at a lower temperature. A small fraction, around $20 \%$, of the TMA molecules are not paired up, as seen in the middle of Fig. 4a (the coverage ratios of the phases shown in Fig. 4 a are not representative). We note that further annealing (up to $420 \mathrm{~K}$ was investigated) did not change the structures.

Whereas deposition of TMA at room temperature led to a disordered phase we now observe perfect order. The ordered 

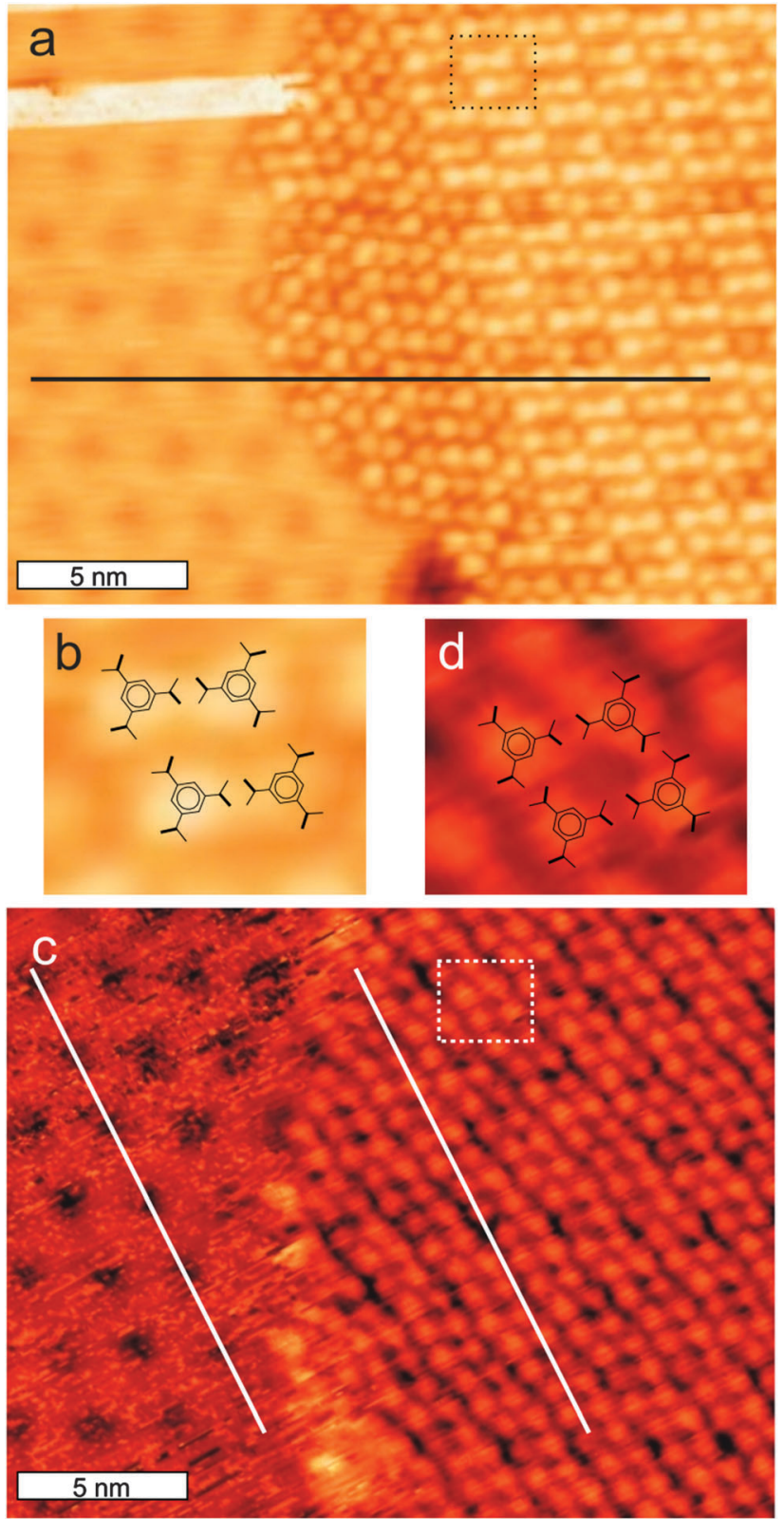

Fig. 4 (a) TMA on $1 \mathrm{ML} \mathrm{Ag/Cu(111)} \mathrm{after} \mathrm{annealing} \mathrm{to} 350 \mathrm{~K}$. The TMA pairs follow the high symmetry orientation of the substrate indicated by the black line. (b) close-up of the area shown by the black dotted rectangle in a. Models of TMA molecules are superimposed. (c) TMA on $3 \mathrm{ML}$ $\mathrm{Ag} / \mathrm{Cu}(111)$ after annealing to $350 \mathrm{~K}$. There is no correlation between the TMA network and the substrate orientation indicated by the white lines. In both images the modulated $(9 \times 9) \mathrm{Ag} / \mathrm{Cu}(111)$ is visible on the left side. (d) close-up of the area shown by the white dotted rectangle in c. Models of TMA molecules are superimposed.

arrangement after annealing is in full agreement with the nature of the intermolecular bonding. TMA in the close-packed arrangement interact via ionic hydrogen bonds that are much stronger than the hydrogen bonds formed between intact TMA molecules upon RT deposition. ${ }^{15,18,19}$ The change in bonding mechanism and binding energy thus alters the balance between molecule-molecule and molecule-surface interaction: at RT deposition the moleculesurface interaction dominates and disturbs the ordering of the self-assembly, whereas annealing and the associated deprotonation leads to an increased intermolecular interaction that reduces the influence from the surface. The modulation the substrate imposes is apparently not strong enough to disorder the assembly of the deprotonated TMA molecules. When we look at the geometry we find that the close-packed phase on $\mathrm{Ag} / \mathrm{Cu}(111)$ is to some extent similar to the one observed on $\mathrm{Ag}(111)$. It experiences a comparable packing density but it is important to note that the symmetry and orientation of the molecular network is now partly steered by the substrate. We find that the long axis of the TMA pairs (horizontal direction in Fig. 4a) is aligned along the $\mathrm{Ag} / \mathrm{Cu}(111)$ Moiré.

We also investigated the self-assembly of TMA on multilayered $\mathrm{Ag}$ on $\mathrm{Cu}(111)$. The modulation of the substrate induced by the reconstruction is still present, but the apparent corrugation gradually decreases from $0.25 \AA$ at $1 \mathrm{ML}$ to $0.15 \AA$ at $3 \mathrm{ML} \mathrm{Ag}$ as we add more layers of silver. Note that these numbers will depend on how sharp the STM tip is. Sub-monolayer amounts of TMA deposited on $3 \mathrm{ML} \mathrm{Ag} / \mathrm{Cu}(111)$ at RT forms dominantly honey-comb structures like on $\mathrm{Ag}(111)$ and annealing to $350 \mathrm{~K}$ leads again to the closepacked structure consisting only of TMA pairs (Fig. 4c), but there is no correlation between the substrate and the orientation of the TMA molecules. Although the modulation is still present on $3 \mathrm{ML} \mathrm{Ag} /$ $\mathrm{Cu}(111)$ it appears to be too small to change the self-assembly as it was observed on $1 \mathrm{ML} \mathrm{Ag}$, where the modulation induced disorder in the molecular structure at room temperature and forced the TMA network to partly follow the modulation upon annealing.

\section{Conclusions}

We have investigated the self-assembly of TMA on the flat surface of $\mathrm{Cu}(111)$ and the modulated surface of $\mathrm{Ag} / \mathrm{Cu}(111)$. Comparisons to previous results on flat $\mathrm{Ag}(111)$ were made. TMA deposited at room temperature on $\mathrm{Cu}(111)$ self-assembles into the same open honeycomb networks as on $\mathrm{Ag}(111)$. A mild annealing to $325 \mathrm{~K}$ leads to a close-packed ordered phase that is distinct from $\mathrm{Ag}(111)$ in terms of a higher packing density and a different molecular arrangement. Room temperature adsorption of TMA on the modulated $\mathrm{Ag} / \mathrm{Cu}(111)$ surface results in a molecular arrangement without long range order since the modulation of the surface disturbs the ordering of the TMA. Annealing the surface to $350 \mathrm{~K}$ transforms the molecular adlayer into a highly ordered close-packed phase that is partly steered by the substrate symmetry. We rationalize that TMA in the close-packed arrangement interact via ionic hydrogen bonds that are much stronger than the hydrogen bonds formed between intact TMA molecules upon RT deposition. This recovers the long range order. We have thus demonstrated how it is possible to switch the balance between molecule-molecule vs. molecule-surface interaction and the associated effects on the self-assembly.

\section{References}

1 T. Yokoyama, S. Yokoyama, T. Kamikado, Y. Okuno and S. Mashiko, Nature, 2001, 413, 619.

2 J.-M. Lehn, Proc. Natl. Acad. Sci. U. S. A., 2002, 99, 4763. 
3 J. V. Barth, G. Costantini and K. Kern, Nature, 2005, 437, 671 .

4 J. V. Barth, Annu. Rev. Phys. Chem., Annual Reviews, Palo Alto, 2007, vol. 58, p. 375.

5 E. Umbach, K. Glöckler and M. Sokolowski, Surf. Sci., 1998, 402-404, 20.

6 R. Fink, D. Gador, U. Stahl, Y. Zou and E. Umbach, Phys. Rev. B: Condens. Matter Mater. Phys., 1999, 60, 2818.

7 T. Suzuki, T. Lutz, D. Payer, N. Lin, S. L. Tait, G. Costantini and K. Kern, Phys. Chem. Chem. Phys., 2009, 11, 6498.

8 S. Lukas, S. Vollmer, G. Witte and C. Wöll, J. Chem. Phys., 2001, 114, 10123.

9 N. Lin, A. Langner, S. L. Tait, C. Rajadurai, M. Ruben and K. Kern, Chem. Commun., 2007, 4860.

10 J. Mao, H. Zhang, Y. Jiang, Y. Pan, M. Gao, W. Xiao and H. J. Gao, J. Am. Chem. Soc., 2009, 131, 14136.

11 H. Zhou, L. Zhang, J. Mao, G. Li, Y. Zhang, Y. Wang, S. Du, W. Hofer and H.-J. Gao, Nano Res., 2013, 6, 131.

12 H. Brune, Surf. Sci. Rep., 1998, 31, 125.

13 A. Dmitriev, N. Lin, J. Weckesser, J. V. Barth and K. Kern, J. Phys. Chem. B, 2002, 106, 6907.

14 N. Lin, D. Payer, A. Dmitriev, T. Strunskus, C. Wöll, J. V. Barth and K. Kern, Angew. Chem., Int. Ed., 2005, 44, 1488.

15 D. Payer, A. Comisso, A. Dmitriev, T. Strunskus, N. Lin, C. Wöll, A. DeVita, J. V. Barth and K. Kern, Chem. - Eur. J., 2007, 13, 3900.

16 Y. Ye, W. Sun, Y. Wang, X. Shao, X. Xu, F. Cheng, J. Li and K. Wu, J. Phys. Chem. C, 2007, 111, 10138.

17 F. Cicoira, C. Santato and F. Rosei, in Stm and Afm Studies on (bio)molecular systems: Unravelling the nanoworld, ed. P. Samori, Springer-Verlag Berlin, Berlin, 2008, vol. 285, p. 203.
18 D. Braga, A. Angeloni, E. Tagliavini and F. Grepioni, J. Chem. Soc., Dalton Trans., 1998, 1961.

19 M. Meot-Ner, Chem. Rev., 2005, 105, 213.

20 L. Kanninen, N. Jokinen, H. Ali-Loytty, P. Jussila, K. Lahtonen, M. Hirsimaki, M. Valden, M. Kuzmin, R. Parna and E. Nommiste, Surf. Sci., 2011, 605, 1968.

21 E. Bauer, Surf. Sci., 1967, 7, 351.

22 K. A. R. Mitchell, D. P. Woodruff and G. W. Vernon, Surf. Sci., 1974, 46, 418.

23 B. Aufray, M. Göthelid, J.-M. Gay, C. Mottet, E. Landemark, G. Falkenberg, L. Lottermoser, L. Seehofer and R. L. Johnson, Microsc., Microanal., Microstruct., 1997, 8, 167.

24 A. Bendounan, H. Cercellier, Y. Fagot-Revurat, B. Kierren, V. Y. Yurov and D. Malterre, Phys. Rev. B: Condens. Matter Mater. Phys., 2003, 67, 165412.

25 U. Müller, D. Carnal, H. Siegenthaler, E. Schmidt, W. J. Lorenz, W. Obretenov, U. Schmidt, G. Staikov and E. Budevski, Phys. Rev. B: Condens. Matter Mater. Phys., 1992, 46, 12899.

26 N. Nilius, E. D. L. Rienks, H.-P. Rust and H.-J. Freund, Phys. Rev. Lett., 2005, 95, 066101.

27 M. Pivetta, F. Patthey, M. Stengel, A. Baldereschi and W.-D. Schneider, Phys. Rev. B: Condens. Matter Mater. Phys., 2005, 72, 115404.

28 A. T. N'Diaye, S. Bleikamp, P. J. Feibelman and T. Michely, Phys. Rev. Lett., 2006, 97, 215501.

29 J. Bork, P. Wahl, L. Diekhöner and K. Kern, New J. Phys., 2009, 11, 113051.

30 J. Bork, J. Onsgaard and L. Diekhöner, J. Phys.: Condens. Matter, 2010, 22, 135005.

31 S. Griessl, M. Lackinger, M. Edelwirth, M. Hietschold and W. M. Heckl, Single Mol., 2002, 3, 25. 